\title{
COMPOSIÇÃO E DISTRIBUIÇÃO ESPAÇO-TEMPORAL DE ANUROS NO PARQUE NATURAL MUNICIPAL AUGUSTO RUSCHI
}

\author{
Matheus de Toledo Moroti ${ }^{1}$ \\ Jonatas Alves Ferreira Neto ${ }^{2}$ \\ Andriele Cristina dos Santos ${ }^{3}$ \\ Mariana Pedrozo 4 \\ Iberê Farina Machado 5 \\ Cristina Pacheco Soares ${ }^{6}$
}

Resumo: Este trabalho teve como objetivo geral avaliar a riqueza de espécies de anuros determinando a sua distribuição espacial e sua sazonalidade. A área de estudo compreende o Parque Natural Municipal Augusto Ruschi, em São José dos Campos (SP). Foram registradas 33 espécies de anuros. A maior riqueza de espécies encontradas vocalizando foi na estação chuvosa e quente, no entanto em relação as análises estatísticas, apenas a temperatura máxima e média mensal apresentaram resultados significativos na riqueza de espécies. As espécies foram encontradas distribuídas em diferentes micro-hábitats e macrohábitats, mostrando que o PNMAR possui uma heterogeneidade ambiental. Os resultados aqui apresentados podem ser utilizados para o manejo do PNMAR, a fim de criar estratégias de preservação dos diferentes habitats de reprodução.

Palavras-chave: Anfíbios; Organização de comunidades; Hábitat; Riqueza de espécies; Reprodução.

\footnotetext{
1 Universidade do Vale do Paraíba. São José dos Campos, São Paulo, Brasil. E-mail: mmoroti@gmail.com.

2 Universidade do Vale do Paraíba. São José dos Campos, São Paulo, Brasil. E-mail: jonatasafn@gmail.com.

3 Universidade do Vale do Paraíba. São José dos Campos, São Paulo, Brasil. E-mail: drieleb2@hotmail.com.

${ }^{4}$ Universidade Federal de Ouro Preto. Ouro Preto, Minas Gerais, Brasil. E-mail: mariana.pedrozo.24@gmail.com.

5 Instituto Boitatá. Goiânia, Goiás, Brasil. E-mail: iberemachado@gmail.com.

${ }^{6}$ Universidade do Vale do Paraíba. São José dos Campos, São Paulo, Brasil. E-mail: cpsoares@univap.br.
} 